\title{
AVALIAÇÃO DE CLONES DE BANANEIRA DO SUBGRUPO CAVENDISH (Musa acuminata, AAA) EM CRUZ DAS ALMAS-BA
}

\author{
Evaluation of banana Cavendish clones in Cruz das Almas City, Bahia State, Brazil \\ Sebastião de Oliveira e Silva ${ }^{1}$, Élio José Alves ${ }^{2}$, Torquato Martins de Andrade Neto ${ }^{3}$, \\ Luis Alberto Lichtemberg ${ }^{4}$, Francisco Ricardo Ferreira ${ }^{5}$
}

\section{RESUMO}

Foram avaliados em Cruz das Almas-BA, em dois ciclos, 69 clones Cavendish coletados em São Paulo, Minas Gerais, Bahia e Santa Catarina, sendo 49 do tipo Nanicão e 20 do tipo Grande Naine. Os clones foram estabelecidos em área experimental da Embrapa Mandioca e Fruticultura utilizando-se um delineamento em blocos ao acaso com cinco repetições e cinco plantas por parcela. Foram utilizados a irrigação por microaspersão, o espaçamento de 3,0 m x 2,0 m e um sistema de condução com três plantas/touceira. Os requerimentos agronômicos foram aplicados conforme preconizados para o cultivo. Na avaliação dos clones, foram consideradas as seguintes variáveis: altura da planta em centímetros (AP); número de dias do plantio à colheita (ND); peso do cacho em quilogramas (PC); número de frutos por cacho (NF); e comprimento do fruto em centímetros (CF). Pelos resultados obtidos, verificou-se uma variabilidade média em todas as variáveis avaliadas. Por apresentarem caracteres superiores, foram selecionados cinco clones de Grande Naine (G.N. Taperão, G.N.Rossete, G.N. Willians, G.N. Magário, G.N. SC-074) e quatro de Nanicão (N. IAC Abóbada Verde, N. Rossete, N. SC-0008 e N. SC-063).

Termos para indexação: Melhoramento, Musa sp, variabilidade, mutações, variedades.

\begin{abstract}
Sixty-nine clones of Cavendish type bananas (49 Nanicão and 20 Grande Naine) collected in the States of São Paulo, Minas Gerais, Bahia and Santa Catarina were evaluated. The clones were established in an experimental area at the Cassava and Fruit Crops Research Center - EMBRAPA, using micro-aspersion irrigation, in plants spaced at $3.0 \mathrm{~m}$ x $2.0 \mathrm{~m}$, and conducted with three plants/bushes. Random blocks were used with five replications and five plants per plot. All cultural requirements for the crop were carried out. For the clone evaluations, the following variables were considered: plant height in centimeters (PH); number of days from planting to yield (ND); weight of bunch in kilograms (WB); number of fruits per bunch (FB) and length of fruit in centimeters (LF). The results obtained showed medium variability in all the characteristics evaluated and five clones of Grande Naine (G.N. Taperão, G.N.Rossete, G.N. Willians, G.N. Magário, G.N. SC-074) and four of Nanicão (N. IAC Green Vault, N. Rossete, N. SC-0008 and N. SC-063) were selected for presenting superior characteristics.
\end{abstract}

Index terms: Musa sp, breeding, variability, mutations, varieties.

(Recebido para publicação em $1^{\circ}$ de outubro de 2003 e aprovado em 8 de outubro de 2004)

\section{INTRODUÇÃO}

Conhecer a situação dos recursos fitogenéticos de um país ou região é da maior importância para se avaliar acessos, resolver sinonímia, identificar mutantes e classificar cultivares de acordo com o genoma, utilizando-se critérios morfotaxonômicos. No caso de fruteiras, e especialmente banana, atenção especial deve ser prestada à prospecção, coleta e conservação de clones, para evitar a erosão da reserva genética e assegurar sua utilização nos programas de melhoramento (SINGH e CHADLA, 1996).
A aquisição de germoplasma promissor introduzido de outras regiões pode atingir os mesmos propósitos de um programa de melhoramento para obtenção de variedades superiores. Assim, a introdução é considerada como um método de melhoramento, visto que fornece a variabilidade genética necessária para a obtenção de cultivares e/ou seleção de clones (ALLARD, 1971).

$\mathrm{Na}$ bananeira, ocorrem variações somaclonais em nível muito superior ao que se observa na maioria das outras culturas, provavelmente devido à instabilidade mitótica, que não é exclusiva da cultura de tecidos, sendo também observada em campo, porém, em freqüências menores (HWANG e TANG, 1996). Dessa

1. Engenheiro Agrônomo, D.Sc., Pesquisador da Embrapa Mandioca e Fruticultura, Caixa Postal 007, 44380-000, Cruz das Almas, BA, ssilva@cpmnf.embrapa.br

2. Engenheiro Agrônomo, M.Sc., Pesquisador Aposentado da Embrapa Mandioca e Fruticultura.

3. Estudante de Agronomia/UFBA.

4. Engenheiro Agrônomo, M.Sc., Pesquisador da EPAGRI, Caixa Postal 277, 88034-901, Itajaí, SC.

5. Engenheiro Agrônomo, D.Sc., Pesquisador da Embrapa Recursos Genéticos e Biotecnologia, Caixa Postal 02372, 70770-900, Brasília, DF. 
forma, pode ser explicado o aparecimento de dezenas de cultivares do grupo genômico (AAA), subgrupo Cavendish, e de cultivares AAB, como a 'Pacovan', mutante da 'Prata'. Mediante a avaliação de germoplasma, pode-se obter ganhos de produtividade e resistência/tolerância a pragas e doenças, a exemplo das cultivares (AAB) Pacovan, Prata Anã, Caipira, Thap Maeo e Nam e dos hìbridos (AAAB) FHIA-18 e SH3640, já recomendados aos agricultores e FHIA 21 (plátano) a ser lançado (SILVA et al., 2002a).

Em Cuba, a seleção baseada em variação somaclonal, resultante do cultivo de tecidos de banana, tem gerado avanços genéticos apreciáveis no melhoramento de bananeira, com recomendação de novas cultivares (RODRIGUEZ-NODALS et al., 1991).

O futuro do melhoramento e/ou manutenção da cadeia produtiva da banana implica não só na exploração da diversidade de tipos de cultivares na geração de híbridos, mas também do uso da variabilidade genética dentro dos diversos clones (SHEPHERD, 1994). Assim, cada variedade de banana tem suas próprias vantagens e desvantagens e pode gerar mais ou menos variantes.

Os produtores devem levar em conta que a solução para seus problemas de produção podem não residir nos genótipos precisamente melhorados. Os enfoques holísticos de manejo integrado dos cultivos também têm muito o que oferecer (DANIELLS, 2000).

A coleta e a avaliação de germoplasma de bananeira do tipo Cavendish apresentam-se, no Brasil, como alternativas para obtenção de novos materiais, visando à utilização como variedades nos sistemas de produção.

Objetivou-se com este trabalho avaliar o desempenho de clones de bananeira do subgrupo Cavendish coletados em diferentes locais do País, nas condições edafoclimáticas de Cruz das Almas-BA.

\section{MATERIAL E MÉTODOS}

Foram avaliados em Cruz das Almas-BA 69 clones de Cavendish coletados em áreas de agricultores, nos Estados de São Paulo, Minas Gerais, Bahia e Santa Catarina (Tabela 1), sendo 49 do tipo Nanicão e 20 do tipo Grande Naine (FERREIRA e SILVA, 2002). A maioria dos clones usada constituía seleção realizada pelos próprios agricultores ao longo dos anos. Entre o material coletado, alguns eram provenientes de mudas micropropagadas e outros resultaram de propagação convencional. A avaliação foi realizada em área experi- mental da Embrapa Mandioca e Fruticultura, usando mudas convencionais sob irrigação por microaspersão, no espaçamento 3,0 m x 2,0 m, e o plantio, conduzido com três plantas por touceira.

O solo da área experimental é um Latossolo Amarelo Álico A moderado, textura franco argiloarenosa, fase de transição floresta tropical subperenifolia/subcadusifolia, com declive de 0 a 3\% (EMBRAPA, 1991).

O experimento foi instalado em maio de 2000 em delineamento de blocos ao acaso, com 69 tratamentos (clones de Cavendish), cinco repetições de cinco touceiras cada uma e com bordadura externa contornando a área experimental. As médias foram analisadas pelo Teste de Scott \& Knott a $5 \%$ de probabilidade.

Os requerimentos agronômicos relativos ao preparo do solo, calagem, abertura de covas, adubação, plantio, práticas culturais e tratamentos fitossanitários foram realizados conforme preconizados para o cultivo da bananeira na região de Cruz das Almas-BA (ALVES e OLIVEIRA, 1999; BORGES et al., 1999).

Foram consideradas as seguintes variáveis na avaliação dos referidos clones em dois ciclos de produção: altura da planta em centímetros (AP); número de dias do plantio à colheita (ND); peso do cacho em quilogramas (PC); número de frutos por cacho (NF); e comprimento do fruto em centímetros (CF).

\section{RESULTADOS E DISCUSSÃO}

Na Tabela 2 são apresentados as médias de altura de planta e o número de dias do plantio à colheita do primeiro e segundo ciclos e a média desses ciclos. A altura da planta, no primeiro ciclo, variou de 253,7 cm, no clone N. SC-072, a 164,0 cm, no clone G.N. Nanica, e no segundo ciclo, variou de $312,0 \mathrm{~cm}$, no clone N. SC-056, a 160,0 cm, no clone N.Cruvinel I. Na média dos dois ciclos, a característica variou de 255,3 cm, no clone N. SC-008, a 172,0 cm, no clone G.N. Nanica. De uma forma geral, os clones Grande Naine apresentaram um porte menor do que os clones Nanicão, como era de se esperar. Embora um dos objetivos da coleta de cultivares Cavendish tenha sido a obtenção de plantas com menor porte, a maioria dos clones avaliados apresentou altura superior a 2,00 m no primeiro ciclo e 2,40 m no segundo ciclo. Dessa forma, os resultados para essa variável foram superiores aos observados por Silva et al. (2002b), que encontraram altura de planta de $1,96 \mathrm{~m}$ e $2,17 \mathrm{~m}$ no primeiro e segundo ciclos, respectivamente, para a cultivar Grande Naine. 
TABELA 1 - Acessos de germoplasma de banana coletados nos Estados de São Paulo, Minas Gerais, Bahia e Santa Catarina.

\begin{tabular}{|c|c|c|c|c|}
\hline Nome $^{1}$ & Município & Latitude & Longitude & Altitude \\
\hline G.N. C. Baixo & Nova Porteirinha-MG & $15^{\circ} 47^{\prime} \mathrm{S}$ & $43^{\circ} 20^{\prime} \mathrm{W}$ & 516 \\
\hline G.N. Cobalchini & Nova Porteirinha-MG & $15^{\circ} 47^{\prime} \mathrm{S}$ & $43^{\circ} 20^{\prime} \mathrm{W}$ & 516 \\
\hline G.N. Colares & Montes Claros-MG & $16^{\circ} 13^{\prime} \mathrm{S}$ & $43^{\circ} 58^{\prime} \mathrm{W}$ & 535 \\
\hline G.N. F. Israel & Registro-SP & $24^{\circ} 38^{\prime} \mathrm{S}$ & $47^{\circ} 54^{\prime} \mathrm{W}$ & 20 \\
\hline G.N. Fernandes & Avaré-SP & $23^{\circ} 06^{\prime} \mathrm{S}$ & $48^{\circ} 57^{\prime} \mathrm{W}$ & 670 \\
\hline G.N. Franco & Registro-SP & $24^{\circ} 38^{\prime} \mathrm{S}$ & $47^{\circ} 54^{\prime} \mathrm{W}$ & 20 \\
\hline G.N. Graneira & Pirapora-MG & $17^{\circ} 26^{\prime} \mathrm{S}$ & $44^{\circ} 42^{\prime} \mathrm{W}$ & 500 \\
\hline G.N. Kinachita & Pirapora-MG & $17^{\circ} 22^{\prime} \mathrm{S}$ & $44^{\circ} 51^{\prime} \mathrm{W}$ & 495 \\
\hline G.N. Leão & João Pinheiro-MG & $17^{\circ} 25^{\prime} \mathrm{S}$ & $46^{\circ} 08^{\prime} \mathrm{W}$ & 775 \\
\hline G.N. M. Jana & Janáuba-MG & $15^{\circ} 16^{\prime} \mathrm{S}$ & $43^{\circ} 24^{\prime} \mathrm{W}$ & 510 \\
\hline G.N. Magário & Sete Barras-SP & $24^{\circ} 21^{\prime} \mathrm{S}$ & $47^{\circ} 56^{\prime} \mathrm{W}$ & 25 \\
\hline G.N. Nanica & Cruz das Almas-BA & $12^{\circ} 44^{\prime} \mathrm{S}$ & $39^{\circ} 06^{\prime} \mathrm{W}$ & 220 \\
\hline G.N. Ribeiro & Nova Porteirinha-MG & $15^{\circ} 46^{\prime} \mathrm{S}$ & $43^{\circ} 18^{\prime} \mathrm{W}$ & 505 \\
\hline G.N. Rossete & Jacupiranga-SP & $24^{\circ} 45^{\prime} \mathrm{S}$ & $48^{\circ} 00^{\prime} \mathrm{W}$ & 25 \\
\hline G.N. SC-064 & Miracatú-SP & $24^{\circ} 16^{\prime} \mathrm{S}$ & $47^{\circ} 28^{\prime} \mathrm{W}$ & 27 \\
\hline G.N. SC-074 & Miracatú-SP & $24^{\circ} 16^{\prime} \mathrm{S}$ & $47^{\circ} 28^{\prime} \mathrm{W}$ & 27 \\
\hline G.N. Taperão & Brotas-SP & $22^{\circ} 14^{\prime} \mathrm{S}$ & $48^{\circ} 13^{\prime} \mathrm{W}$ & 520 \\
\hline G.N. Valery & Cruz das Almas-BA & $12^{\circ} 44^{\prime} \mathrm{S}$ & $39^{\circ} 06^{\prime} \mathrm{W}$ & 220 \\
\hline G.N. Williams & Cruz das Almas-BA & $12^{\circ} 44^{\prime} \mathrm{S}$ & $39^{\circ} 06^{\prime} \mathrm{W}$ & 220 \\
\hline Grande Naine & Cruz das Almas-BA & $12^{\circ} 44^{\prime} \mathrm{S}$ & $39^{\circ} 06^{\prime} \mathrm{W}$ & 220 \\
\hline N. A. Verde & Pariquera-Açu-SP & $24^{\circ} 44^{\prime} \mathrm{S}$ & $47^{\circ} 56^{\prime} \mathrm{W}$ & 20 \\
\hline N. Bentinho & Cajati-SP & $24^{\circ} 47^{\prime} \mathrm{S}$ & $48^{\circ} 08^{\prime} \mathrm{W}$ & 30 \\
\hline N. Canela & Cruz das Almas-BA & $12^{\circ} 44^{\prime} \mathrm{S}$ & $39^{\circ} 06^{\prime} \mathrm{W}$ & 220 \\
\hline N. Colares & Montes Claros-MG & $16^{\circ} 13^{\prime} S$ & $43^{\circ} 58^{\prime} \mathrm{W}$ & 535 \\
\hline N. Cruvinel I & Paracatu-MG & $17^{\circ} 15^{\prime} \mathrm{S}$ & $46^{\circ} 40^{\prime} \mathrm{W}$ & 600 \\
\hline N. Cruvinel II & Paracatu-MG & $17^{\circ} 15^{\prime} \mathrm{S}$ & $46^{\circ} 40^{\prime} \mathrm{W}$ & 600 \\
\hline \multirow[t]{2}{*}{ N. Damásio } & Janáuba-MG & $15^{\circ} 39^{\prime} \mathrm{S}$ & $43^{\circ} 33^{\prime} \mathrm{W}$ & 520 \\
\hline & & & & Continue \\
\hline
\end{tabular}


TABELA 1 - Continuação...

\begin{tabular}{|c|c|c|c|c|}
\hline Nome $^{1}$ & Município & Latitude & Longitude & Altitude \\
\hline N. Fernandes & Avaré-SP & $23^{\circ} 06^{\prime} \mathrm{S}$ & $48^{\circ} 57^{\prime} \mathrm{W}$ & 670 \\
\hline N. Franco & Registro-SP & $24^{\circ} 38^{\prime} S$ & $47^{\circ} 54^{\prime} \mathrm{W}$ & 20 \\
\hline N. Grande Naine & Registro-SP & $24^{\circ} 38^{\prime} \mathrm{S}$ & $47^{\circ} 54^{\prime} \mathrm{W}$ & 20 \\
\hline N. Huber & Paranapanema-SP & $23^{\circ} 23^{\prime} \mathrm{S}$ & $48^{\circ} 49^{\prime} \mathrm{W}$ & 650 \\
\hline N. Iida & Pirapora-MG & $17^{\circ} 28^{\prime} S$ & $44^{\circ} 50^{\prime} \mathrm{W}$ & 490 \\
\hline N. J. Beckers & Paranapanema-SP & $23^{\circ} 28^{\prime} \mathrm{S}$ & $48^{\circ} 41^{\prime} \mathrm{W}$ & 635 \\
\hline N. J. Leme & Registro-SP & $24^{\circ} 34^{\prime} \mathrm{S}$ & $47^{\circ} 52^{\prime} \mathrm{W}$ & 20 \\
\hline N. Lima & Capitão Enéas-MG & $16^{\circ} 12^{\prime} \mathrm{S}$ & $43^{\circ} 51^{\prime} \mathrm{W}$ & 530 \\
\hline N. M. Verde I & Verdelândia-MG & $15^{\circ} 35^{\prime} \mathrm{S}$ & $43^{\circ} 30^{\prime} \mathrm{W}$ & 520 \\
\hline N. M. Verde II & Verdelândia-MG & $15^{\circ} 35^{\prime} \mathrm{S}$ & $43^{\circ} 30^{\prime} \mathrm{W}$ & 520 \\
\hline N. Magário & Cruz das Almas-BA & $12^{\circ} 44^{\prime} \mathrm{S}$ & $39^{\circ} 06^{\prime} \mathrm{W}$ & 220 \\
\hline N. Oiola & Nova Porteirinha-MG & $15^{\circ} 48^{\prime} \mathrm{S}$ & $43^{\circ} 21^{\prime} \mathrm{W}$ & 515 \\
\hline N. Olin & Avandu-SP & $23^{\circ} 08^{\prime} \mathrm{S}$ & $49^{\circ} 02^{\prime} \mathrm{W}$ & 660 \\
\hline N. Ribeiro & Nova Porteirinha-MG & $15^{\circ} 46^{\prime} \mathrm{S}$ & $43^{\circ} 18^{\prime} \mathrm{W}$ & 505 \\
\hline N. Rossete & Jacupiranga-SP & $24^{\circ} 45^{\prime} \mathrm{S}$ & $48^{\circ} 00^{\prime} \mathrm{W}$ & 25 \\
\hline N. Souza & Nova Porteirinha-MG & $15^{\circ} 45^{\prime} \mathrm{S}$ & $43^{\circ} 19^{\prime} \mathrm{W}$ & 505 \\
\hline N. Veloso & Paracatu-MG & $17^{\circ} 12^{\prime} \mathrm{S}$ & $46^{\circ} 34^{\prime} \mathrm{W}$ & 600 \\
\hline N. Yamada & Janáuba-MG & $15^{\circ} 47^{\prime} \mathrm{S}$ & $43^{\circ} 23^{\prime} \mathrm{W}$ & 515 \\
\hline Nanicão & Cruz das Almas-BA & $12^{\circ} 44^{\prime} \mathrm{S}$ & $39^{\circ} 06^{\prime} \mathrm{W}$ & 220 \\
\hline Naniquinha. B & Registro-SP & $24^{\circ} 34^{\prime} \mathrm{S}$ & $47^{\circ} 52^{\prime} \mathrm{W}$ & 20 \\
\hline N. SC-006 & Criciúma-SC & $28^{\circ} 41^{\prime} \mathrm{S}$ & $49^{\circ} 22^{\prime} \mathrm{W}$ & 46 \\
\hline N. SC-007 & Siderópolis-SC & $28^{\circ} 35^{\prime} \mathrm{S}$ & $49^{\circ} 26^{\prime} \mathrm{W}$ & 147 \\
\hline N. SC-008 & Jacinto Machado-SC & $29^{\circ} 00^{\prime} \mathrm{S}$ & $49^{\circ} 46^{\prime} \mathrm{W}$ & 50 \\
\hline N. SC-018 & Palhoça-SC & $27^{\circ} 40^{\prime} \mathrm{S}$ & $48^{\circ} 40^{\prime} \mathrm{W}$ & 3 \\
\hline N. SC-019 & Itajaí-SC & $27^{\circ} 00^{\prime} \mathrm{S}$ & $48^{\circ} 45^{\prime} \mathrm{W}$ & 1 \\
\hline N. SC-023 & Porto Belo-SC & $26^{\circ} 10^{\prime} \mathrm{S}$ & $48^{\circ} 30^{\prime} \mathrm{W}$ & 1 \\
\hline N. SC-027 & Tijucas-SC & $27^{\circ} 15^{\prime} \mathrm{S}$ & $48^{\circ} 40^{\prime} \mathrm{W}$ & 2 \\
\hline
\end{tabular}


TABELA 1 - Continuação...

\begin{tabular}{|c|c|c|c|c|}
\hline Nome $^{1}$ & Município & Latitude & Longitude & Altitude \\
\hline N. SC-031 & Itajaí-SC & $27^{\circ} 00^{\prime} S$ & $48^{\circ} 45^{\prime} \mathrm{W}$ & 1 \\
\hline N. SC-032 & Garuva-SC & $26^{\circ} 05^{\prime} \mathrm{S}$ & $48^{\circ} 50^{\prime} \mathrm{W}$ & 25 \\
\hline N. SC-033 & Garuva-SC & $26^{\circ} 00^{\prime} S$ & $48^{\circ} 50^{\prime} \mathrm{W}$ & 25 \\
\hline N. SC-043 & Corupá-SC & $26^{\circ} 25^{\prime} \mathrm{S}$ & $49^{\circ} 10^{\prime} \mathrm{W}$ & 62 \\
\hline N. SC-056 & Pariquera-Açú-SP & $24^{\circ} 44^{\prime} \mathrm{S}$ & $47^{\circ} 56^{\prime} \mathrm{W}$ & 20 \\
\hline N. SC-063 & Miracatú-SP & $24^{\circ} 16^{\prime} \mathrm{S}$ & $47^{\circ} 28^{\prime} \mathrm{W}$ & 27 \\
\hline N. SC-072 & Itajaí-SC & $27^{\circ} 00^{\prime} \mathrm{S}$ & $48^{\circ} 45^{\prime} \mathrm{W}$ & 1 \\
\hline N. SC-073 & Camboriú-SC & $27^{\circ} 15^{\prime} \mathrm{S}$ & $48^{\circ} 38^{\prime} \mathrm{W}$ & 8 \\
\hline N. SC-078 & Corupá-SC & $26^{\circ} 25^{\prime} \mathrm{S}$ & $49^{\circ} 10^{\prime} \mathrm{W}$ & 62 \\
\hline N. SC-079 & Corupá-SC & $26^{\circ} 25^{\prime} \mathrm{S}$ & $49^{\circ} 10^{\circ} \mathrm{W}$ & 62 \\
\hline N. SC-080 & Corupá-SC & $26^{\circ} 25^{\prime} \mathrm{S}$ & $49^{\circ} 10^{\prime} \mathrm{W}$ & 62 \\
\hline N. SC-082 & Guaramirim-SC & $26^{\circ} 27^{\prime} \mathrm{S}$ & $49^{\circ} 00^{\prime} \mathrm{W}$ & 30 \\
\hline N. SC-087 & Corupá-SC & $26^{\circ} 25^{\prime} \mathrm{S}$ & $49^{\circ} 10^{\prime} \mathrm{W}$ & 62 \\
\hline N. SC-098 & Corupá-SC & $26^{\circ} 25^{\prime} \mathrm{S}$ & $49^{\circ} 10^{\prime} \mathrm{W}$ & 62 \\
\hline N. SC-099 & Corupá-SC & $26^{\circ} 25^{\prime} \mathrm{S}$ & $49^{\circ} 10^{\prime} \mathrm{W}$ & 62 \\
\hline
\end{tabular}

${ }^{1}$ N:Nanicão; GN: Grande Naine.

O clone G.N. Leão, com o ciclo total de 387,0 dias, foi o mais tardio, enquanto o clone mais precoce foi o N. Franco, que apresentou um ciclo total de 324,7 dias no primeiro ciclo. Já no segundo ciclo, o clone mais tardio foi o N. Cluvinel ll, com o ciclo total de 706,8 dias, enquanto o clone mais precoce, o G.N. Graneira, apresentou um ciclo total de 585,6 dias. Em termos de média do número de dias do plantio à colheita, observou-se uma variação de 535,1 (G.N. Leão) a 461,7 (N. SC-043). O ciclo é um carácter de relevância no melhoramento genético da bananeira, já que um menor ciclo reflete a precocidade da planta e a redução do número de dias compreendidos entre o plantio e a colheita.

O peso de cacho, o número e o comprimento dos frutos do primeiro e segundo ciclos e a média desses são apresentados na Tabela 3.

O peso do cacho obteve uma variação de $29,0 \mathrm{~kg}$ (G.N. Rossete) a 13,0 kg (G.N. Leão) no primeiro ciclo, ao passo que, no segundo ciclo, variou de 35,0 kg (G.N. M. Jana) a 13,0 kg (Grande Naine). A média do peso do cacho variou de 29,5 kg (G.N. Magário) a 17,5 kg (Grande Naine). Os clones Grande Naine, em geral, apresentaram maior peso do cacho do que os clones Nanicão, no primeiro ciclo, embora o genótipo com menor peso do cacho tenha sido observado em um clone Grande Naine. O mesmo não ocorreu no segundo ciclo, em que a predominância de cachos mais pesados foi dos clones Nanicão.

À exceção dos clones G.N. Cobalchini e G.N. Leão, que apresentaram, no primeiro ciclo, peso de cacho de $15,0 \mathrm{~kg}$ e $13,0 \mathrm{~kg}$, respectivamente, todos os demais apresentaram valores para essa característica superiores ao obtido (15,6 kg) por Silva et al. (2002b). No segundo ciclo, os clones G.N. M. Jana e N. Ribeiro, com cachos de $35,0 \mathrm{~kg}$ e $32,5 \mathrm{~kg}$, produziram mais que o dobro do valor observado $(15,9 \mathrm{~kg})$ pelos mesmos autores. Na média dos dois ciclos, a produção variou de $29,5 \mathrm{~kg}$ (G.N. Magário) a 17,5 (Grande Naine). Em ambos os ciclos, houve a formação de apenas dois agrupamentos com aplicação de Teste de Scott \& Knott. Provavelmente, o alto CV impediu uma melhor discriminação de grupos de clones com relação ao peso do cacho (PC), levando-se a indicação de uma baixa variabilidade com relação a essa característica. 
TABELA 2 - Médias de altura de planta (AP) em cm e do número de dias do plantio à colheita (ND) no primeiro e segundo ciclos de 69 genótipos de bananeira em Cruz das Almas-BA ${ }^{1}$.

\begin{tabular}{|c|c|c|c|c|c|c|}
\hline \multirow{3}{*}{ Genótipos } & \multicolumn{6}{|c|}{ Variáveis } \\
\hline & \multicolumn{3}{|c|}{ Altura da Planta } & \multicolumn{3}{|c|}{ Número de Dias } \\
\hline & $1^{o}$ ciclo & $2^{o}$ ciclo & Média & $1^{0}$ ciclo & $2^{o}$ ciclo & Média \\
\hline N. SC-072 & 253,7 a & 240,0 c & 246,8 & $336,0 \mathrm{~b}$ & $607,6 \mathrm{c}$ & 471,8 \\
\hline N. Canela & 238,0 a & $250,0 \mathrm{c}$ & 244,0 & $345,0 \mathrm{~b}$ & $635,2 \mathrm{~b}$ & 490,1 \\
\hline N. SC-032 & 236,0 a & $260,0 \mathrm{c}$ & 248,0 & 347,7 b & $625,2 \mathrm{~b}$ & 486,4 \\
\hline N. SC-027 & $235,0 \mathrm{a}$ & $270,0 \mathrm{~b}$ & 252,5 & $350,0 \mathrm{~b}$ & $634,6 \mathrm{~b}$ & 492,3 \\
\hline N. SC-082 & 233,0 a & $220,0 \mathrm{~d}$ & 226,5 & 348,7 b & $634,2 \mathrm{~b}$ & 491,4 \\
\hline N. Souza & $232,0 \mathrm{a}$ & $240,0 \mathrm{c}$ & 236,0 & $337,0 \mathrm{~b}$ & 609,4 c & 473,2 \\
\hline N. A. Verde & $231,0 \mathrm{a}$ & 260,0 c & 245,5 & 358,7 a & $646,0 \mathrm{~b}$ & 502,3 \\
\hline N. SC-018 & $230,0 \mathrm{a}$ & 180,0 e & 205,0 & $335,0 \mathrm{~b}$ & $592,8 \mathrm{c}$ & 463,9 \\
\hline N. M. Verde I & 227,7 a & 180,0 e & 203,3 & $350,0 \mathrm{~b}$ & 623,2 c & 486,6 \\
\hline N. Ribeiro & $223,0 \mathrm{a}$ & 254,0 c & 238,5 & $361,0 \mathrm{a}$ & 609,6 c & 485,3 \\
\hline N. Magário & 223,0 a & $242,0 \mathrm{c}$ & 232,5 & 369,7 a & 626,2 b & 497,9 \\
\hline N. J. Leme & 222,7 a & $240,0 \mathrm{c}$ & 216,0 & $350,0 \mathrm{~b}$ & $592,0 \mathrm{c}$ & 471,0 \\
\hline N. SC-033 & $222,0 \mathrm{a}$ & 260,0 c & 241,0 & $335,0 \mathrm{~b}$ & 611,6 c & 483.3 \\
\hline N. SC-019 & $222,0 \mathrm{a}$ & $280,0 \mathrm{a}$ & 251,0 & 356,0 a & $625,6 \mathrm{~b}$ & 490,8 \\
\hline N. SC-073 & $222,0 \mathrm{a}$ & $200,0 \mathrm{~d}$ & 211,0 & 342,7 b & 621,6 c & 482.1 \\
\hline N. Bentinho & $222,0 \mathrm{a}$ & $280,0 \mathrm{~b}$ & 251,0 & $355,0 \mathrm{a}$ & $635,2 \mathrm{~b}$ & 495,1 \\
\hline N. Fernandes & $221,0 \mathrm{a}$ & 250,0 c & 235,5 & 348,7 b & 629,2 b & 485,9 \\
\hline N. SC-008 & $220,7 \mathrm{a}$ & 290,0 a & 255,3 & $343,0 \mathrm{~b}$ & 615,0 c & 479,0 \\
\hline N. SC-080 & 220,7 a & $260,0 \mathrm{c}$ & 240,3 & $357,0 \mathrm{a}$ & 616,2 c & 484,6 \\
\hline N. Franco & 220,0 a & 236,0 c & 228,0 & 324,7 a & 613,2 c & 468,9 \\
\hline N. SC-063 & 220,0 a & $272,0 \mathrm{~b}$ & 246,0 & 355,7 a & 601,8 c & 478,7 \\
\hline N. Rossete & $219,7 \mathrm{a}$ & $251,0 \mathrm{c}$ & 235,3 & $336,0 \mathrm{~b}$ & $600,0 \mathrm{c}$ & 468,0 \\
\hline N. SC-078 & 218,7 a & 260,0 c & 239,3 & 350,7 b & $621,8 \mathrm{c}$ & 486,2 \\
\hline N. J. Beckers & 218,7 a & $236,0 \mathrm{c}$ & 231,3 & 344,7 b & $626,2 \mathrm{~b}$ & 485.4 \\
\hline N. Huber & 218,7 a & $240,0 \mathrm{c}$ & 229,3 & 344,7 b & 622,2 c & 483,4 \\
\hline
\end{tabular}


TABELA 2 - Continuação...

\begin{tabular}{|c|c|c|c|c|c|c|}
\hline \multirow{3}{*}{ Genótipos } & \multicolumn{6}{|c|}{ Variáveis } \\
\hline & \multicolumn{3}{|c|}{ Altura da Planta } & \multicolumn{3}{|c|}{ Número de Dias } \\
\hline & $1^{0}$ ciclo & $2^{o}$ ciclo & Média & $1^{o}$ ciclo & $2^{\circ}$ ciclo & Média \\
\hline G.N. Valery & $218,0 \mathrm{a}$ & $270,0 \mathrm{~b}$ & 242,0 & $345,0 \mathrm{~b}$ & $623,6 \mathrm{~b}$ & 484,3 \\
\hline N. SC-043 & 217,0 a & 260,0 c & 238,5 & $330,0 \mathrm{~b}$ & 593,4 c & 461,7 \\
\hline N. SC-031 & 216,0 a & $260,0 \mathrm{c}$ & 238,0 & 359,7 a & 608,0 c & 483.8 \\
\hline N. SC-006 & $215,0 \mathrm{a}$ & $270,0 \mathrm{~b}$ & 242,5 & 341,7 b & 594,8 c & 468,2 \\
\hline N. SC-007 & $215,0 \mathrm{a}$ & 260,0 c & 237,5 & $360,0 \mathrm{a}$ & $632,2 \mathrm{~b}$ & 496,1 \\
\hline Nanicão & $213,0 \mathrm{a}$ & $250,0 \mathrm{c}$ & 226,5 & $340,7 \mathrm{~b}$ & $640,8 \mathrm{~b}$ & 490,7 \\
\hline N. Iida & $212,0 \mathrm{a}$ & $220,0 \mathrm{~d}$ & 222,3 & 358,0 a & $638,4 \mathrm{~b}$ & 498,2 \\
\hline N. Olin & 211,7 a & 250,0 c & 230,8 & 351,7 a & $625,0 \mathrm{~b}$ & 488,3 \\
\hline G.N. Rossete & $211,0 \mathrm{a}$ & $230,0 \mathrm{~d}$ & 220,5 & $342,0 \mathrm{~b}$ & $623,0 \mathrm{c}$ & 482,5 \\
\hline G.N. Fernandes & 206,7 a & 250,0 c & 228,3 & $329,0 \mathrm{~b}$ & 608,6 c & 468,6 \\
\hline G.N. M. Jana & 206,7 a & 260,0 c & 233,3 & 352,7 a & $625,6 \mathrm{~b}$ & 489,1 \\
\hline N. SC-056 & 206,0 a & $312,0 \mathrm{a}$ & 209,0 & 354,0 a & 597,6 c & 475,8 \\
\hline N. SC-087 & 206,0 a & 250,0 c & 228,0 & 330,7 b & 599,8 c & 465,2 \\
\hline N. Oiola & 206,0 a & 250,0 c & 228,0 & 361,7 a & 609,0 c & 485,3 \\
\hline G.N. F. Israel & $203,0 \mathrm{~b}$ & $220,0 \mathrm{~d}$ & 211,5 & $348,0 \mathrm{~b}$ & $646,2 \mathrm{~b}$ & 497,1 \\
\hline N. Damásio & $203,0 \mathrm{~b}$ & $250,0 \mathrm{c}$ & 226,5 & $369,7 \mathrm{a}$ & $649,8 \mathrm{~b}$ & 509,7 \\
\hline G.N. Graneira & $202,0 \mathrm{~b}$ & $226,0 \mathrm{~d}$ & 214,0 & 355,0 a & 585,6 c & 470,3 \\
\hline N. Colores & 201,7 b & 240,0 c & 220,8 & $345,0 \mathrm{~b}$ & $635,8 \mathrm{~b}$ & 490,4 \\
\hline Nanicão. G.N. & $201,0 \mathrm{~b}$ & $262,0 \mathrm{c}$ & 231,5 & $340,0 \mathrm{~b}$ & 623,2 b & 481.6 \\
\hline N. SC-099 & $201,0 \mathrm{~b}$ & $220,0 \mathrm{~d}$ & 210,5 & $352,0 \mathrm{a}$ & 619,6 c & 485,8 \\
\hline G.N. Kinachita & $201,0 \mathrm{~b}$ & $200,0 \mathrm{~d}$ & 200,5 & 361,7 a & 673,6 a & 517,6 \\
\hline G.N. Magário & 200,7 b & $210,0 \mathrm{~d}$ & 205,3 & $342,0 \mathrm{~b}$ & 619,0 c & 480,5 \\
\hline Grande Naine & $200,0 \mathrm{~b}$ & $230,0 \mathrm{~d}$ & 215,0 & 353,0 a & $641,0 \mathrm{~b}$ & 497,0 \\
\hline G.N. Williams & 196,7 b & $240,0 \mathrm{c}$ & 218,3 & 354,7 a & 634,6 b & 494,6 \\
\hline \multirow[t]{2}{*}{ G.N. Ribeiro } & $196,0 \mathrm{~b}$ & 256,0 c & 226,0 & $328,0 \mathrm{~b}$ & 613,0 c & 470,5 \\
\hline & & & & & & Contir \\
\hline
\end{tabular}


TABELA 2 - Continuação...

\begin{tabular}{|c|c|c|c|c|c|c|}
\hline \multirow{3}{*}{ Genótipos } & \multicolumn{6}{|c|}{ Variáveis } \\
\hline & \multicolumn{3}{|c|}{ Altura da Planta } & \multicolumn{3}{|c|}{ Número de Dias } \\
\hline & $1^{0}$ ciclo & $2^{\circ}$ ciclo & Média & $1^{\circ}$ ciclo & $2^{\circ}$ ciclo & Média \\
\hline G.N. SC-064 & $196,0 \mathrm{~b}$ & $220,0 \mathrm{~d}$ & 208,0 & 366,0 a & $657,8 \mathrm{~b}$ & 511,9 \\
\hline N. Cruvinel I & 195,7 b & 160,0 e & 177,8 & 357,7 a & 653,2 b & 505,4 \\
\hline G.N. Franco & $192,0 \mathrm{~b}$ & $220,0 \mathrm{~d}$ & 206,0 & $342,0 \mathrm{~b}$ & 619,2 с & 480,6 \\
\hline G.N. SC-074 & $192,0 \mathrm{~b}$ & $224,0 \mathrm{~d}$ & 208,0 & 340,7 b & $628,2 \mathrm{~b}$ & 484,4 \\
\hline N. Yamada & 191,7 b & $240,0 \mathrm{c}$ & 215,8 & 334,7 b & $645,4 \mathrm{~b}$ & 490,1 \\
\hline G.N. Taperão & 189,7 b & $220,0 \mathrm{~d}$ & 209,8 & 350,7 b & $647,4 \mathrm{~b}$ & 499,1 \\
\hline G.N. Cobalchini & $186,7 \mathrm{~b}$ & $250,0 \mathrm{c}$ & 218,3 & 385,0 a & $674,4 \mathrm{a}$ & 529,7 \\
\hline N. SC-079 & 183,0 c & $250,0 \mathrm{c}$ & 216,5 & 354,0 a & 613,2 a & 483,6 \\
\hline N. Veloso & 181,7 c & $260,0 \mathrm{c}$ & 220,8 & 368,7 a & $682,6 \mathrm{a}$ & 525,6 \\
\hline G.N. Leão & 181,7 c & $220,0 \mathrm{~d}$ & 205,3 & 387,0 a & $683,2 \mathrm{a}$ & 535,1 \\
\hline N. SC-023 & 179,0 c & 258,0 c & 218,5 & $342,0 \mathrm{~b}$ & 642,2 b & 492,1 \\
\hline G.N. Colares & 175,0 c & $210,0 \mathrm{~d}$ & 192,5 & 357,0 a & 598,2 c & 477,6 \\
\hline N. SC-098 & 174,7 c & 257,0 c & 216,5 & 374,7 a & $637,8 \mathrm{~b}$ & 506,2 \\
\hline N. Lima & 171,7 c & $246,0 \mathrm{c}$ & 208,8 & 381,0 a & $644,0 \mathrm{~b}$ & 512,5 \\
\hline N. Cruvinel II & 169,0 с & $230,0 \mathrm{~d}$ & 199,5 & 346,0 b & 706,8 a & 526,4 \\
\hline Naniquinha. B & 167,7 c & 190,0 e & 178,8 & 341,7 b & 614,0 c & 477,8 \\
\hline N. M. Verde II & 167,0 c & $260,0 \mathrm{c}$ & 213,0 & 355,0 a & $634,0 \mathrm{~b}$ & 494,5 \\
\hline G.N. C. Baixo & 165,0 c & 180,0 e & 172,5 & 336,7 b & $638,0 \mathrm{~b}$ & 487,3 \\
\hline G.N. Nanica & 164,0 c & 180,0 e & 172,0 & 356,0 a & 631,2 b & 493,6 \\
\hline CV\% & 7,676 & 8,635 & & 4,926 & 5,815 & \\
\hline
\end{tabular}

${ }^{1}$ Médias seguidas pela mesma letra nas colunas não diferem significativamente entre si pelo Teste de Scott \& Knott, a 5\% de probabilidade. 
Avaliação de clones de bananeira do subgrupo Cavendish...

TABELA 3 - Médias do peso de cacho (PC), do número de frutos (NF) e do comprimento de frutos (CF) no primeiro e segundo ciclos de 69 genótipos de bananeira em Cruz das Almas-BA ${ }^{1}$.

\begin{tabular}{|c|c|c|c|c|c|c|c|c|c|}
\hline \multirow{3}{*}{ Genótipos } & \multicolumn{9}{|c|}{ Variáveis } \\
\hline & \multicolumn{3}{|c|}{ Peso de Cacho } & \multicolumn{3}{|c|}{ Número de Frutos } & \multicolumn{3}{|c|}{ Comprimento de Fruto } \\
\hline & $1^{\circ}$ ciclo & $2^{\circ}$ ciclo & Média & $1^{\circ}$ ciclo & $2^{\circ}$ ciclo & Média & $1^{\circ}$ ciclo & $2^{\circ}$ ciclo & Média \\
\hline N. SC-072 & $21,0 \mathrm{~b}$ & 23,6 a & 22,7 & $131,0 \mathrm{~b}$ & $152,4 \mathrm{a}$ & 141,7 & 19,0 a & $19,0 \mathrm{~b}$ & 19,0 \\
\hline N. Canela & $17,3 \mathrm{~b}$ & 24,6 a & 20,9 & $121,7 \mathrm{~b}$ & $147,2 \mathrm{a}$ & 134,5 & $17,0 \mathrm{a}$ & 19,5 a & 18,2 \\
\hline N. SC-032 & 26,0 a & 24,4 a & 25,2 & 143,0 a & $153,8 \mathrm{a}$ & 148,4 & $20,0 \mathrm{a}$ & 19,5 a & 19,7 \\
\hline N. SC-027 & $23,0 \mathrm{a}$ & 25,5 a & 24,8 & 140,7 a & $152,4 \mathrm{a}$ & 146,5 & $18,0 \mathrm{a}$ & $20,0 \mathrm{a}$ & 19,0 \\
\hline N. SC-082 & 26,0 a & 28,6 a & 27,3 & $144,0 \mathrm{a}$ & $154,2 \mathrm{a}$ & 149,1 & $20,0 \mathrm{a}$ & 19,7 a & 19,7 \\
\hline N. Souza & 21,7 a & 23,4 a & 23,0 & 147,7 a & $157,0 \mathrm{a}$ & 152,3 & 18,0 a & 18,2 b & 18,1 \\
\hline N. A. Verde & 26,0 a & 31,4 a & 28,7 & 142,7 a & $172,0 \mathrm{a}$ & 157,4 & 19,0 a & $19,0 \mathrm{~b}$ & 19,0 \\
\hline N. SC-018 & $23,0 \mathrm{a}$ & 24,4 a & 23,7 & $120,0 \mathrm{~b}$ & 156,8 a & 138,4 & 20,7 a & $17,8 \mathrm{c}$ & 18,9 \\
\hline N. M. Verde I & 23,9 a & 24,5 a & 24,2 & $132,0 \mathrm{~b}$ & $154,6 \mathrm{a}$ & 143,3 & 19,0 a & 18,9 b & 18,9 \\
\hline N. Ribeiro & $18,0 \mathrm{~b}$ & 32,5 a & 25,3 & $117,0 \mathrm{~b}$ & $172,2 \mathrm{a}$ & 144,5 & 19,0 a & 20,4 a & 19,7 \\
\hline N. Magário & $20,0 \mathrm{~b}$ & 25,4 a & 22,7 & $131,0 \mathrm{~b}$ & 154,8 a & 142,6 & 18,7 a & $19,0 \mathrm{~b}$ & 18,8 \\
\hline N. J. Leme & 23,0 a & 26,9 a & 24,9 & $142,0 \mathrm{a}$ & $131,8 \mathrm{~b}$ & 136,8 & 19,0 a & 20,8 a & 18,8 \\
\hline N. SC-033 & $25,0 \mathrm{a}$ & 27,4 a & 26,2 & 164,7 a & $162,0 \mathrm{a}$ & 163,3 & 19,0 a & 19,5 a & 19,2 \\
\hline N. SC-019 & $19,0 \mathrm{~b}$ & $20,6 \mathrm{~b}$ & 19,8 & $117,7 \mathrm{~b}$ & $130,2 \mathrm{~b}$ & 143,6 & 18,7 a & $18,4 \mathrm{~b}$ & 18,5 \\
\hline N. SC-073 & $22,0 \mathrm{a}$ & $20,7 \mathrm{~b}$ & 21,3 & $143,0 \mathrm{a}$ & $130,2 \mathrm{~b}$ & 136,6 & 19,0 a & $18,9 \mathrm{~b}$ & 18,9 \\
\hline N. Bentinho & $20,0 \mathrm{~b}$ & $18,0 \mathrm{~b}$ & 19,0 & $132,0 \mathrm{~b}$ & $137,8 \mathrm{~b}$ & 134,9 & 18,0 a & $17,0 \mathrm{c}$ & 17,5 \\
\hline N. Fernandes & 23,0 a & $18,8 \mathrm{~b}$ & 20,9 & $130,7 \mathrm{~b}$ & $137,6 \mathrm{~b}$ & 135,2 & 19,0 a & 16,2 c & 17,6 \\
\hline N. SC-008 & 23,0 a & 26,0 a & 24,5 & 149,7 a & $135,6 \mathrm{~b}$ & 142,8 & 20,0 a & $20,0 \mathrm{a}$ & 20,0 \\
\hline N. SC-080 & $21,0 \mathrm{~b}$ & 28,6 a & 24,8 & $110,0 \mathrm{~b}$ & $161,6 \mathrm{a}$ & 135,8 & 20,0 a & $21,0 \mathrm{a}$ & 20,5 \\
\hline N. Franco & $24,0 \mathrm{a}$ & 25,0 a & 24,5 & 155,0 a & $132,0 \mathrm{~b}$ & 143,5 & 19,7 a & 19,9 a & 19,8 \\
\hline N. SC-063 & 23,7 a & $20,4 \mathrm{~b}$ & 22,0 & $132,0 \mathrm{~b}$ & $144,8 \mathrm{~b}$ & 138,4 & 19,0 a & 19,4 a & 19,2 \\
\hline N. Rossete & $26,0 \mathrm{a}$ & 28,6 a & 27,3 & $140,0 \mathrm{a}$ & 156,2 a & 148,1 & 20,0 a & 20,3 a & 20,1 \\
\hline N. SC-078 & $22,0 \mathrm{a}$ & 27,9 а & 24,9 & $152,0 \mathrm{a}$ & 155,2 a & 153,6 & 19,0 a & 21,0 a & 20,0 \\
\hline N. J. Beckers & $19,0 \mathrm{~b}$ & $19,5 \mathrm{~b}$ & 19,2 & $131,0 \mathrm{~b}$ & 158,4 a & 144,7 & 19,0 a & 18,8 b & 19,9 \\
\hline N. Huber & $22,0 \mathrm{a}$ & $25,2 \mathrm{a}$ & 23,6 & $126,0 \mathrm{~b}$ & $135,2 \mathrm{~b}$ & 130,6 & 19,0 a & 19,5 a & 22,7 \\
\hline G.N. Valery & 24,0 a & 25,6 a & 24,8 & $152,0 \mathrm{a}$ & $150,6 \mathrm{a}$ & 151,3 & 20,0 a & 19,9 a & 19,9 \\
\hline N. SC-043 & $23,0 \mathrm{a}$ & $28,2 \mathrm{a}$ & 25,6 & 145,0 a & 156,6 a & 150,8 & $20,0 \mathrm{a}$ & $20,0 \mathrm{a}$ & 20,0 \\
\hline
\end{tabular}


TABELA 3 - Continuação...

\begin{tabular}{|c|c|c|c|c|c|c|c|c|c|}
\hline \multirow{3}{*}{ Genótipos } & \multicolumn{9}{|c|}{ Variáveis } \\
\hline & \multicolumn{3}{|c|}{ Peso de Cacho } & \multicolumn{3}{|c|}{ Número de Frutos } & \multicolumn{3}{|c|}{ Comprimento de Fruto } \\
\hline & $1^{o}$ ciclo & $2^{\circ}$ ciclo & Média & $1^{o}$ ciclo & $2^{\circ}$ ciclo & Média & $1^{\circ}$ ciclo & $2^{\circ}$ ciclo & Média \\
\hline N. SC-031 & $21,0 \mathrm{~b}$ & 31,7 a & 26,3 & $127,0 \mathrm{~b}$ & 171,4 a & 149,2 & $18,0 \mathrm{a}$ & 20,7 a & 19,3 \\
\hline N. SC-006 & $24,0 \mathrm{a}$ & 29,6 a & 26,8 & $133,0 \mathrm{~b}$ & 178,0 a & 155,5 & $19,0 \mathrm{a}$ & 19,2 a & 19,1 \\
\hline N. SC-007 & $20,0 \mathrm{~b}$ & 30,6 a & 25,3 & $125,0 \mathrm{~b}$ & 179,8 a & 152,4 & 19,0 a & 20,6 a & 19,8 \\
\hline Nanicão & 24,0 a & $25,1 \mathrm{a}$ & 24,5 & $146,0 \mathrm{a}$ & 164,8 a & 155,4 & 19,0 a & 19,3 a & 19,2 \\
\hline N. Iida & $19,0 \mathrm{~b}$ & $24,8 \mathrm{a}$ & 21,9 & 143,0 a & 173,4 a & 158,2 & $19,0 \mathrm{a}$ & $18,7 \mathrm{~b}$ & 18,9 \\
\hline N. Olin & 24,0 a & 28,2 a & 26,1 & $122,0 \mathrm{~b}$ & 172,8 a & 147,4 & $20,0 \mathrm{a}$ & 20,8 a & 20,4 \\
\hline G.N. Rossete & $29,0 \mathrm{a}$ & $25,0 \mathrm{a}$ & 27,0 & $146,0 \mathrm{a}$ & 154,0 a & 150,0 & $20,0 \mathrm{a}$ & 20,6 a & 20,3 \\
\hline G.N. Fernandes & 22,7 a & 29,2 a & 25,9 & 156,7 a & 159,8 a & 158,2 & 19,0 a & $20,2 \mathrm{a}$ & 19,6 \\
\hline G.N. M. Jana & $24,0 \mathrm{a}$ & 35,0 a & 26,9 & 135,7 b & 180,0 a & 157,8 & $20,0 \mathrm{a}$ & $20,0 \mathrm{a}$ & 20,0 \\
\hline N. SC-056 & $17,0 \mathrm{~b}$ & $25,4 \mathrm{a}$ & 21,2 & $125,0 \mathrm{~b}$ & 163,0 a & 144,0 & $18,0 \mathrm{a}$ & 19,5 a & 18,7 \\
\hline N. SC-087 & $19,0 \mathrm{~b}$ & 26,0 a & 22,5 & 137,0 a & $139,6 \mathrm{~b}$ & 138,3 & 19,0 a & 20,2 a & 19,6 \\
\hline N. Oiola & $19,0 \mathrm{~b}$ & 24,8 a & 21,9 & $119,0 \mathrm{~b}$ & 150,2 a & 134,6 & $20,0 \mathrm{a}$ & $18,3 \mathrm{~b}$ & 19,2 \\
\hline G.N. F. Israel & 22,7 a & 24,5 a & 23,6 & 131,7 b & $125,6 \mathrm{~b}$ & 128,6 & 19,0 a & 19,6 a & 19,3 \\
\hline N. Damásio & $17,0 \mathrm{~b}$ & 25,4 a & 21,2 & $110,0 \mathrm{~b}$ & 146,8 a & 128,4 & $18,0 \mathrm{a}$ & 19,6 a & 18,8 \\
\hline G.N. Graneira & $22,0 \mathrm{a}$ & $18,3 \mathrm{~b}$ & 20,1 & $122,0 \mathrm{~b}$ & 120,4 b & 121,2 & $18,0 \mathrm{a}$ & $18,6 \mathrm{~b}$ & 18,3 \\
\hline N. Colores & $22,0 \mathrm{a}$ & $16,5 \mathrm{~b}$ & 19,3 & 150,0 a & 117,4 b & 133,7 & 18,0 a & $18,7 \mathrm{~b}$ & 18,3 \\
\hline N.Grande Naine & 25,0 a & 28,4 a & 25,2 & 149,0 a & 153,8 a & 151,4 & $19,0 \mathrm{a}$ & 19,5 a & 19,2 \\
\hline N. SC-099 & 22,0 a & $19,2 \mathrm{~b}$ & 20,6 & $132,0 \mathrm{~b}$ & $136,0 \mathrm{~b}$ & 134,0 & $19,0 \mathrm{a}$ & $17,1 \mathrm{c}$ & 18,0 \\
\hline G.N. Kinachita & $27,0 \mathrm{a}$ & $13,2 \mathrm{~b}$ & 20,1 & 138,0 a & $92,0 \mathrm{~b}$ & 115,0 & $19,7 \mathrm{a}$ & $18,1 \mathrm{~b}$ & 18,9 \\
\hline G.N. Magário & 27,0 a & 26,9 a & 29,5 & 161,0 a & 161,6 a & 161,3 & $18,7 \mathrm{a}$ & $18,4 \mathrm{~b}$ & 18,5 \\
\hline Grande Naine & 22,0 a & $13,0 \mathrm{~b}$ & 17,5 & 143,0 a & 151,6 a & 147,3 & $18,0 \mathrm{a}$ & 16,7 c & 17,3 \\
\hline G.N. Williams & 24,0 a & 30,5 a & 27,3 & $131,0 \mathrm{~b}$ & 161,6 a & 146,3 & $20,0 \mathrm{a}$ & $19,1 \mathrm{a}$ & 19,5 \\
\hline G.N. Ribeiro & 26,0 a & $25,0 \mathrm{a}$ & 25,5 & 145,0 a & 156,0 a & 150,5 & $19,7 \mathrm{a}$ & $17,4 \mathrm{c}$ & 18,5 \\
\hline G.N. SC-064 & 25,0 a & $21,4 \mathrm{~b}$ & 23,2 & 145,0 a & $133,0 \mathrm{~b}$ & 139,0 & $19,0 \mathrm{a}$ & 19,2 a & 19,1 \\
\hline N. Cruvinel I & $19,0 \mathrm{~b}$ & $20,8 \mathrm{~b}$ & 19,9 & $121,0 \mathrm{~b}$ & 147,8 a & 134,4 & $18,0 \mathrm{a}$ & $18,6 \mathrm{~b}$ & 18,3 \\
\hline G.N. Franco & 24,0 a & $25,2 \mathrm{a}$ & 24,6 & 154,0 a & 148,0 a & 151,0 & $19,0 \mathrm{a}$ & 20,0 a & 19,5 \\
\hline G.N. SC-074 & $24,0 \mathrm{a}$ & $28,0 \mathrm{a}$ & 26,0 & 139,0 a & $139,8 \mathrm{~b}$ & 139,4 & $20,0 \mathrm{a}$ & 20,8 a & 20,4 \\
\hline
\end{tabular}


TABELA 3 - Continuação...

\begin{tabular}{|c|c|c|c|c|c|c|c|c|c|}
\hline \multirow{3}{*}{ Genótipos } & \multicolumn{9}{|c|}{ Variáveis } \\
\hline & \multicolumn{3}{|c|}{ Peso de Cacho } & \multicolumn{3}{|c|}{ Número de Frutos } & \multicolumn{3}{|c|}{ Comprimento de Fruto } \\
\hline & $1^{\circ}$ ciclo & $2^{\circ}$ ciclo & Média & $1^{\circ}$ ciclo & $2^{\circ}$ ciclo & Média & $1^{\circ}$ ciclo & $2^{\circ}$ ciclo & Média \\
\hline N. Yamada & $19,0 \mathrm{~b}$ & 17,7 b & 18,4 & $128,0 \mathrm{~b}$ & $136,0 \mathrm{~b}$ & 132,0 & $17,0 \mathrm{a}$ & $18,5 \mathrm{~b}$ & 17,7 \\
\hline G.N. Taperão & $25,0 \mathrm{a}$ & 29,8 a & 27,4 & 163,0 a & 175,8 a & 169,4 & $20,0 \mathrm{a}$ & 19,7 a & 19,8 \\
\hline G.N. Cobalchini & $15,0 \mathrm{~b}$ & 24,6 a & 19,8 & $111,7 \mathrm{~b}$ & 153,6 a & 132,6 & $18,0 \mathrm{a}$ & $17,6 \mathrm{c}$ & 17,8 \\
\hline N. SC-079 & $20,0 \mathrm{~b}$ & $21,8 \mathrm{~b}$ & 20,9 & $129,7 \mathrm{~b}$ & $136,6 \mathrm{~b}$ & 132,8 & $18,0 \mathrm{a}$ & 19,3 a & 18,6 \\
\hline N. Veloso & $22,0 \mathrm{a}$ & 25,5 a & 23,7 & $118,0 \mathrm{~b}$ & 156,4 a & 137,2 & $19,0 \mathrm{a}$ & 19,8 a & 19,4 \\
\hline G.N. Leão & $13,0 \mathrm{~b}$ & $25,1 \mathrm{a}$ & 19,0 & $95,0 \mathrm{~b}$ & 150,4 a & 122,7 & 17,7 a & $19,4 \mathrm{a}$ & 18,5 \\
\hline N. SC-023 & $26,0 \mathrm{a}$ & 25,2 a & 25,6 & $161,0 \mathrm{a}$ & 172,8 a & 166,9 & $20,0 \mathrm{a}$ & $19,8 \mathrm{a}$ & 19,9 \\
\hline G.N. Colares & $24,0 \mathrm{a}$ & 25,8 a & 24,9 & 139,0 a & 152,6 a & 145,8 & $19,0 \mathrm{a}$ & 20,4 a & 19,7 \\
\hline N. SC-098 & $21,0 \mathrm{~b}$ & 26,8 a & 23,9 & $125,7 \mathrm{~b}$ & 158,4 a & 141,8 & $19,0 \mathrm{a}$ & 19,8 a & 19,4 \\
\hline N. Lima & $17,0 \mathrm{~b}$ & $20,4 \mathrm{~b}$ & 18,7 & $119,0 \mathrm{~b}$ & $122,4 \mathrm{~b}$ & 120,7 & $18,0 \mathrm{a}$ & $20,1 \mathrm{a}$ & 19,0 \\
\hline N. Cruvinel II & $17,0 \mathrm{~b}$ & 27,4 a & 22,2 & 150,0 a & 160,4 a & 155,2 & 16,7 a & 20,2 a & 18,5 \\
\hline Naniquinha. B & $22,0 \mathrm{a}$ & $25,0 \mathrm{a}$ & 23,5 & $158,0 \mathrm{a}$ & $149,2 \mathrm{a}$ & 153,7 & $19,0 \mathrm{a}$ & 19,9 a & 19,4 \\
\hline N. M. Verde II & $22,0 \mathrm{a}$ & 29,3 a & 25,6 & $152,0 \mathrm{a}$ & $161,1 \mathrm{a}$ & 156,5 & $18,0 \mathrm{a}$ & $20,2 \mathrm{a}$ & 19,1 \\
\hline G.N. C. Baixo & $18,0 \mathrm{~b}$ & $25,2 \mathrm{a}$ & 21,6 & 155,7 a & 156,2 a & 155,9 & 16,7 a & $18,6 \mathrm{~b}$ & 17,6 \\
\hline G.N. Nanica & $22,0 \mathrm{a}$ & $16,8 \mathrm{~b}$ & 19,4 & $133,7 \mathrm{~b}$ & $138,2 \mathrm{~b}$ & 135,9 & 18,0 a & $17,6 \mathrm{c}$ & 17,8 \\
\hline CV\% & 20,38 & 24,30 & & 14,04 & 16,86 & & 9,81 & 7,67 & \\
\hline
\end{tabular}

${ }^{1}$ Médias seguidas pela mesma letra nas colunas não diferem significativamente entre si pelo Teste de Scott \& Knott, a $5 \%$ de probabilidade.

O número de frutos (NF) por cacho no primeiro ciclo variou de 164,7, no clone N. SC-033, a 95,0, no clone G.N. Leão, ao passo que no segundo ciclo houve uma variação de 180,0, no clone G.N. M. Jana, a 92,0, no clone G.N. Kinachita. Em média, o NF variou de 169,4, no clone G.N. Taperão, a 115,0, no clone G.N. Kinachita.

Os valores de número de frutos da cultivar Grande Naine obtidos em Cruz das Almas foram de 120 para o primeiro ciclo e de 126 para o segundo ciclo (SILVA et al., 2002b). No primeiro ciclo, 59 clones superaram esses valores e, no segundo ciclo, praticamente todos os clones produziram um maior número de frutos. Provavelmente, o Grande Naine de Cruz das Almas seja inferior à maioria dos clones coletados com relação a essa característica. O fato de o
Grande Naine ter apresentado um maior número de frutos do que o observado por Silva et al. (2002b) se deveu às condições desfavoráveis ao desenvolvimento do material na avaliação feita por esses pesquisadores.

A variação do comprimento do fruto (CF) no primeiro e segundo ciclos foi, respectivamente, de 20,7 cm (N. SC-018) a 16,7 cm (G.N. C. Baixo e N. Cruvinel II) e $21,0 \mathrm{~cm}(\mathrm{~N}$. SC-080) a $16,2 \mathrm{~cm}$ (N. Fernandes). Em média, o CF variou de 22,7 cm (N. Huber) a 17,3 cm (Grande Naine). O CF mais adequado à exportação é $22 \mathrm{~cm}$ (FRUTISSÉRIES, 2000). Como se pode observar, nenhum clone atingiu esse valor. Frutos maiores, no entanto, poderiam ser obtidos caso fossem realizadas a eliminação do coração e a retirada das últimas pencas. O emprego dessas práticas pode levar a um incremento 
do peso do cacho e do diâmetro do fruto (SOTO BALLESTERO, 1992).

Pela facilidade de seleção, a altura de planta tem sido a característica mais explorada no melhoramento usando variantes somaclonais. No entanto, têm sido observadas variantes para precocidade, disposição foliar, tamanho de fruto, rendimento e resistência à doença (HWANG e TANG, 1996).

\section{CONCLUSÃO}

Pelos resultados, verifica-se que existe uma variabilidade média em todas as variáveis avaliadas e que é possível encontrar genótipos resultantes de mutações espontâneas e/ou variação somaclonal que possam se constituir em novas variedades de bananeira.

Por apresentarem caracteres superiores, foram selecionados cinco clones de Grande Naine (G.N. Taperão, G.N.Rossete, G.N. Willians, G.N. Magário, G.N. SC-074) e quatro de Nanicão (N. IAC Abóbada Verde, N. Rossete, N. SC-0008 e N. SC-063).

\section{REFERÊNCIAS BIBLIOGRÁFICAS}

ALVES, E. J.; OLIVEIRA, M. A. Práticas culturais. In: ALVES, E. J. (Org.). A cultura da banana: aspectos técnicos, socioeconômicos e agroindustriais. 2. ed. rev. Brasília: EMBRAPA-SPI; Cruz das Almas: EMBRAPA-CNPMF, 1999. p. 335-352.

ALLARD, R. W. Princípios de melhoramento genético das plantas. São Paulo: Edgard Blucher, 1971. 381 p.

BORGES, A. L.; OLIVEIRA, A. M. G.; SOUZA, L. S. Solos, nutrição e adubação. In: ALVES, E. J. (Org.). A cultura da banana: aspectos técnicos, socioeconômicos e agroindustriais. 2. ed. rev. Brasília: EMBRAPASPI; Cruz das Almas: EMBRAPA-CNPMF, 1999. p. 197-260.

DANIELLS, J. W. Qué variedad de banano debo cultivar? Informusa, Montpellier, v. 9, n. 1, p. 31-33, jun. 2000 .
EMPRESA BRASILEIRA DE PESQUISA AGROPECUÁRIA. Serviço Nacional de Levantamento e Conservação de Solos. Levantamento detalhado dos solos do Centro Nacional de Pesquisa de Mandioca e Fruticultura Tropical. Cruz das Almas, 1991. 126 p. (Boletim de pesquisa, 39).

FERREIRA, F. R.; SILVA, S. O. Collecting banana germplasm from the AAA genomic group/Cavendish subgroup. Crop Breeding and Applied Biotechnology, Londrina, v. 2, n. 3, p. 485-488, 2002.

FRUTISSÉRIES. Banana. Brasília: MI, 2000.

HWANG, S. C.; TANG, C. Y. Somaclonal variation and its use for improving cavendish (AAA desert) bananas in Taiwan. In: NEW FRONTIERS IN RESISTANCE FOR NEMATODE FUSARIUM AND SIGATOKA, 1., 1995, Kuala Lummpur, Malaysia. Proceedings... Montpellier: Inibap, 1996. p. 173-181.

RODRIGUEZ-NODALS, A. A. et al. Avances en el programa de mejoramiento genético del banano y el plátano en el INIVIT en Cuba: un avance de investigación. Informusa, Montpellier, v. 1, n. 1, p. 3-5, dic. 1991.

SHEPHERD, K. Mejoramiento de bananos historia y métodos. Informusa, Montpellier, v. 3, n. 1, p. 10-11, jun. 1994.

SILVA, S. O. et al. Bananeira. In: BRUCKNER, C. H. (Org.). Melhoramento de fruteiras tropicais. Viçosa: UFV, 2002a. v. 1, p. 101-157.

SILVA, S. O.; FLORES, J. C. de O.; LIMA NETO, F. P. Avaliação de cultivares e híbridos de bananeira em quatro ciclos de produção. Pesquisa Agropecuária Brasileira, Brasilia, v. 37, n. 11, p. 1567-1574, 2002 b.

SINGH, H. P.; CHADLA, K. L. Bananos y plátanos em la India. Informusa, Montpellier, v. 5, n. 2, p. 22-25, dic. 1996.

SOTO BALLESTERO, M. Bananos: cultivo y comercialización. 2. ed. San José: Litografia e Imprenta, 1992. $674 \mathrm{p}$. 